\title{
Familial increased serum intestinal alkaline phosphatase: a new variant associated with Gilbert's syndrome
}

A G Lieverse, G G van Essen, G J J Beukeveld, J Gazendam, E C Dompeling, L P ten Kate, S A van Belle, J Weits

\begin{abstract}
Investigation of mild, inherited increased serum alkaline phosphatase activity partially combined with Gilbert's syndrome in one family showed, apart from a normal liver fraction, an intestinal isoenzyme pattern and an extra band in the agar electrophoresis. Analysis by agarose electrophoresis before and after incubation of neuraminidase showed that the extra fraction was an intestinal variant isoenzyme.

The precise genetic background of the two disorders in this family could not be determined from the available data. Abnormal activities of (regular) intestinal alkaline phosphatase isoenzyme caused the increase in serum alkaline phosphatase in the absence of disease.
\end{abstract}

Increased alkaline phosphatase activities are often found during routine laboratory investigations. ${ }^{1}$ Generally this is associated with bone or hepatobiliary disorders. Apart from clinically important conditions, the possibility of an isolated transient ${ }^{2-6}$ or inherited persistent $^{6-14}$ increase in serum alkaline phosphatase activity has to be considered.

A familial, mild, chronic, unconjugated hyperbilirubinemia without an increase in alkaline phosphatase or aminotransferase activities and without (haemolytic) disease, is diagnosed as Gilbert's syndrome. ${ }^{15-17}$

\section{Methods}

Agar electrophoresis of alkaline phosphatase isoenzymes was done according to the method of Sundblad et al, ${ }^{18}$ except that the staining method was done according to Robson et al. ${ }^{19}$ Electrophoresis of alkaline phosphatase was also performed with an improved method on agarose gels with and without neuraminidase to discriminate between the different isoenzymes. Neuraminidase enzymatically removes negatively charged sialic acid from the alkaline phosphatase glycoproteins. Because intestinal alkaline phosphatase isoenzymes do not contain sialic acid the electrophoretic migration does not shift after incubation with neuraminidase, while all other alkaline phosphatase isoenzymes do shift. Non-shifting fractions have always been proved to be intestinal by immunological methods. ${ }^{2021}$ The method was

University Hospital, Groningen, The

Netherlands

Department of

Internal Medicine

A G Lieverse

$G \mathrm{G}$ van Essen

E C Dompeling

J Weits

Laboratory of Clinical

Chemistry

G J J Beukeveld

Department of

Medical Genetics

L P ten Kate

Laboratory of Clinical

Chemistry, Roman

Catholic Hospital,

Groningen, The

Netherlands

J Gazendam

Barger Compascuüm

$S$ A van Belle

Correspondence to:

Dr J Weits, Department of

Internal Medicine

Internal Medicine,

University Hospital

9713 EZ Groningen,

Accepted for publication

21 September 1989

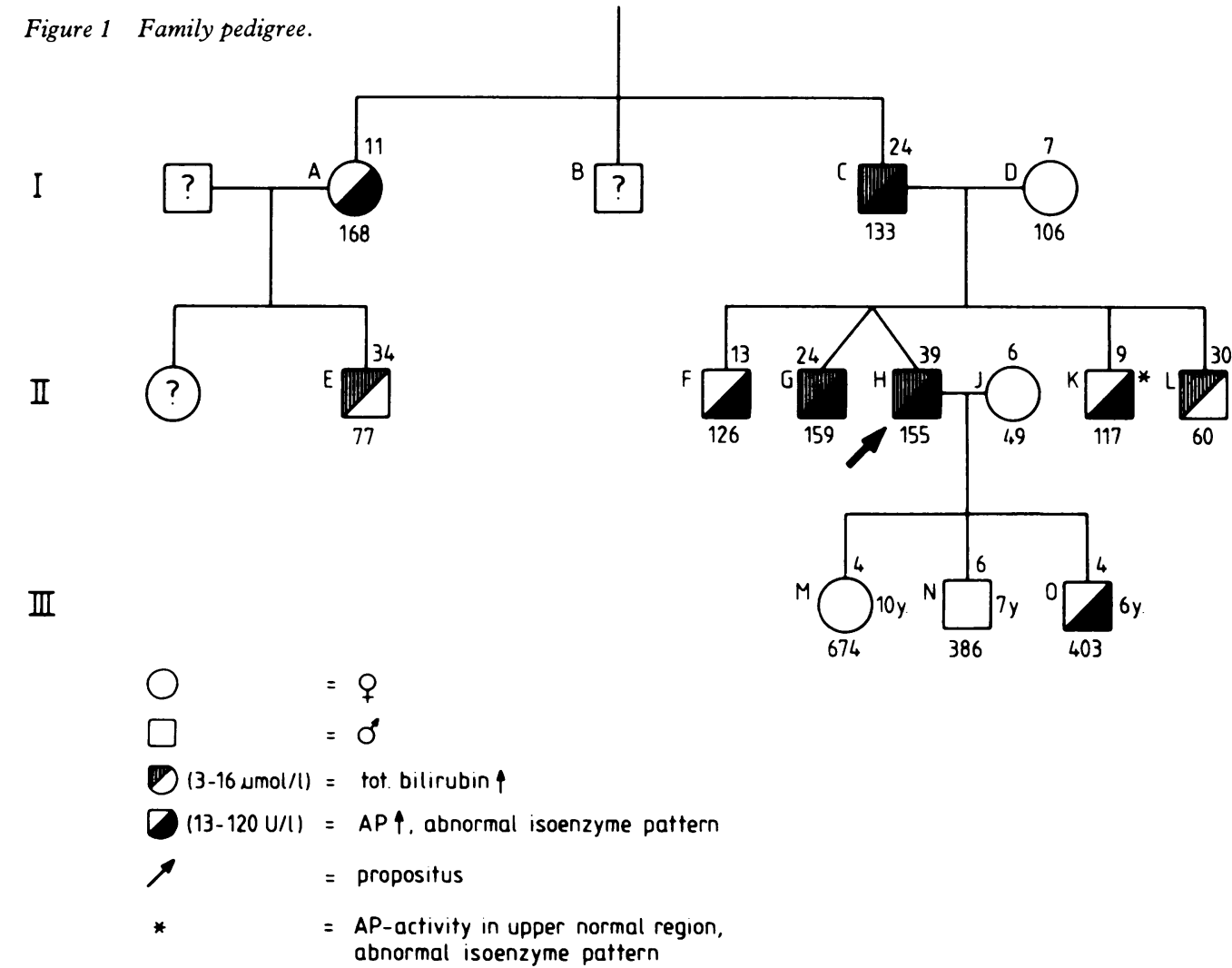




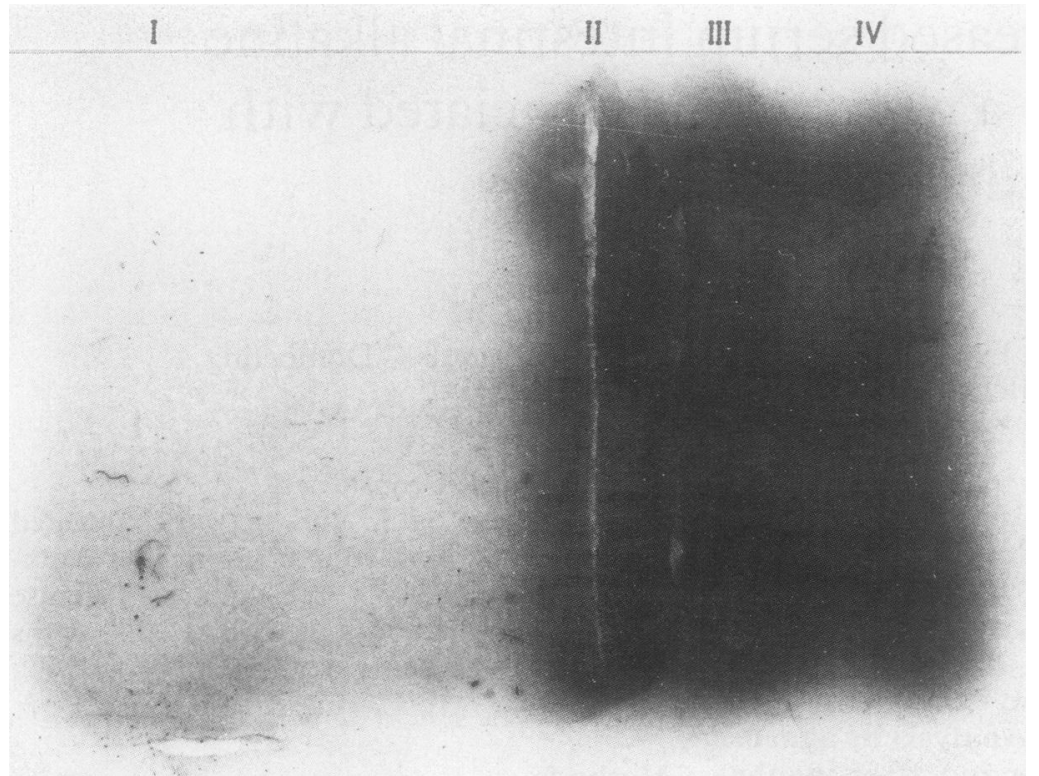

Figure 2 Agar

electrophoresis of alkaline phosphatase.

$I=$ albumin,

$I I=$ application point and liver fraction,

$I I I=$ abnormal fraction

$I V=$ intestinal fraction.

performed according to the manufacturer's instructions (Beckman-Isopal), Analis, Namur, Belgium. After electrophoresis the gels were scanned densitometrically. The absolute activity $(\mathrm{U} / \mathrm{l})$ of each alkaline phosphatase isoenzyme was calculated using the relative distribution of the isoenzymes and the total alkaline phosphatase activity.

\section{CASE REPORT}

A Caucasian man, aged 33, was diagnosed as having chronic persistent hepatitis $B$ after his wife had had acute hepatitis B shortly before (fig 1). Over 20 months we observed a gradual tendency towards normalisation of his transaminase activities: aspartate aminotransferase (AST) decreased from 66 to 32 (normal range 0-40 U/1) and alanine aminotransferase (ALT) from 208 to 61 (normal range 0-30 U/1). Laboratory investigation also showed a persistently increased alkaline phosphatase activity of 155 (normal range 13-120 U/1) and a total bilirubin of 39 (normal range $3-16 \mu \mathrm{mol} / \mathrm{l}$ ) with a conjugated bilirubin of 5 (normal range 0-5 $\mu \mathrm{mol} / \mathrm{l}$ ). These alkaline phosphatase and bilirubin values were thought not to be consistent with the diagnosis of mild chronic hepatitis $B$ infection. Haemolysis was excluded by the finding of normal lactate dehydrogenase activity, haptoglobin concentration, and reticulocyte count. To exclude other hepatobiliary disorders we performed a 99Tc liver scan, which was normal, and a liver biopsy. Microscopy of the biopsy specimen showed chronic persistent hepatitis without cholestasis and with morphologically normal bile ducts. Enzyme histochemical methods showed a normal alkaline phosphatase activity at the hepatocyte membrane. Immunoperoxidase staining showed cytoplasmatic hepatitis $B$ surface antigen ( $\mathrm{HBsAg}$ ) and the absence of hepatitis B core antigen ( $\mathrm{HBcAg}$ ). Agar electrophoresis was done to determine alkaline phosphatase isoenzymes. This showed liver and intestinal isoenzymes and an extra band (fig 2). On account of these findings a family investigation was done.
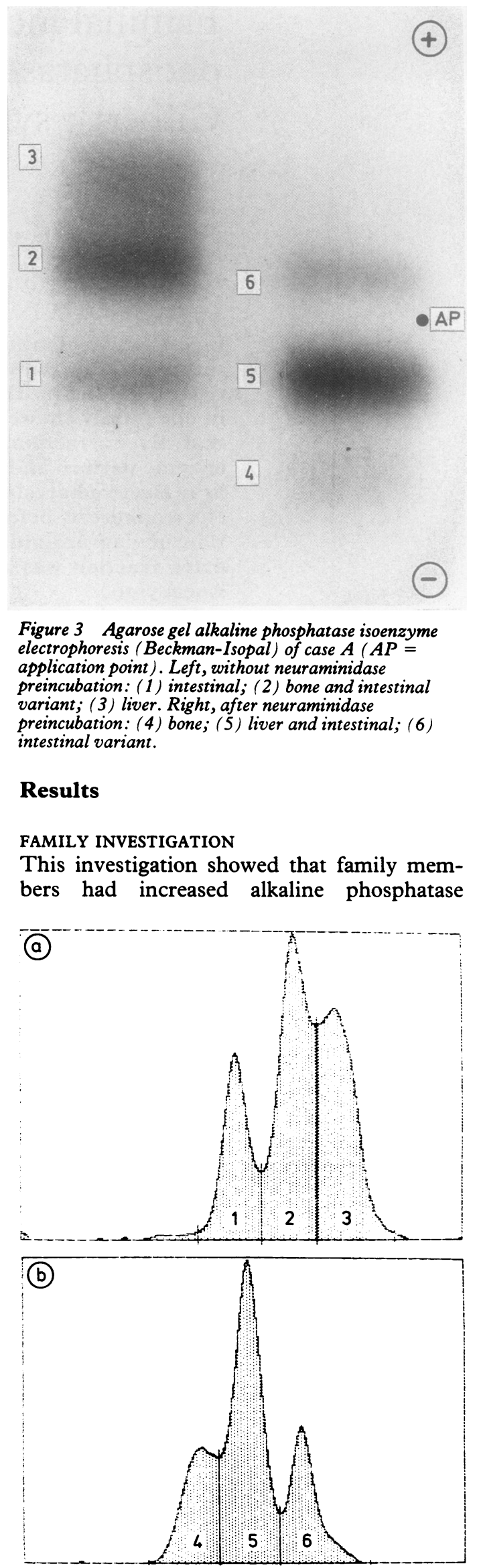

Figure 4 Densitometrical scanning of agarose gel electrophoresis shown in fig 3 with relative fractions $(\%)$. (a) Above, without neuraminidase reincubation: (1) intestinal (22); (2) bone and intestinal variant (46); (3) liver (32). (b) Below, after precincubation with neuraminidase: (4) bone (24); (5) liver and intestinal (54); (6) intestinal variant (22). 
Percentage relative and absolute ( $U / l)$ contribution of alkaline phosphatase isoenzymes to total alkaline phosphatase in some family members with increased alkaline phosphatase

\begin{tabular}{|c|c|c|c|c|c|}
\hline \multirow[b]{2}{*}{ Case No } & \multicolumn{3}{|c|}{ Isoenzyme } & \multirow[b]{2}{*}{ Intestinal variant } & \multirow[b]{2}{*}{ Total alkaline phosphatase } \\
\hline & Bone & Liver & Intestinal & & \\
\hline $\begin{array}{l}\mathrm{A} \\
\mathrm{F} \\
\mathrm{M} \\
\mathrm{K} \\
\mathrm{O}\end{array}$ & $\begin{array}{l}24(40) \\
27(34) \\
40(62) \\
30(35) \\
77(310)\end{array}$ & $\begin{array}{l}32(54) \\
42(52) \\
20(31) \\
40(47) \\
10(40)\end{array}$ & $\begin{array}{c}22(37) \\
18(23) \\
20(31) \\
15(18) \\
4(17)\end{array}$ & $\begin{array}{r}22(37) \\
13(17) \\
20(31) \\
15(18) \\
9(36)\end{array}$ & $\begin{array}{l}168 \mathrm{U} / 1 \\
126 \mathrm{U} / 1 \\
155 \mathrm{U} / 1 \\
117 \mathrm{U} / 1 \\
403 \mathrm{U} / 1\end{array}$ \\
\hline
\end{tabular}

activities, or unconjugated hyperbilirubinemia, or a combination (fig 1). Other liver function tests were normal in all those investigated. Except for mild jaundice during "flulike" illnesses in the index case and his identical twin brother, nobody complained of other symptoms. To exclude post hepatitis B hyperbilirubinaemia in his family full hepatitis B serology was done in all family members and shown to be negative (except in the index case). All those with increased alkaline phosphatase activity except for a 10 year old girl (case $M$, fig 1) and her 7 year old brother (case N, fig 1), had a liver isoenzyme, an intestinal isoenzyme, and an identical extra band in agar electrophoresis. This band and the intestinal band were absent in those with normal alkaline phosphatase activities and also not present in the two children ( $M$ and $N$ ) in whom this could be accounted for by active bone growth. All family members with an abnormal isoenzyme pattern in the agar eletrophoresis seemed to have a normal liver and bone fraction but intestinal and intestinal variant isoenzymes as extra components in the agarose electrophoresis (figs 3 and 4). All family members with a normal alkaline phosphatase isoenzyme pattern in the agar electrophoresis had a normal pattern after analysis in the agarose method (figs 5 and 6).

Calculation of the absolute contribution of alkaline phosphatase isoenzyme fractions gave the following results (table). In adults the bone alkaline phosphatase was between 34 and $62 \mathrm{U} /$ l, liver alkaline phosphatase between 31

Figure 5 Agarose gel alkaline phosphatase isoenzyme electrophoresis (Beckman-Isopal) of case $J$ (fig 1) (normal pattern). Left, without neuraminidase

preincubation: (7a) bone; (7b) liver. Right, after neuraminidase

preincubation: (8) bone; (9) liver. and $54 \mathrm{U} / 1$, intestinal alkaline phosphatase between 17 and $37 \mathrm{U} / \mathrm{l}$ and intestinal variant alkaline phosphatase between 17 and $37 \mathrm{U} / 1$. In all investigated adults the sum of bone alkaline phosphatase and liver alkaline phosphatase was less than $95 \mathrm{U} / 1$, which is normal. Serum intestinal and intestinal variant isoenzymes may be present normally but only in small amounts ( $<10 \mathrm{U} / 1$, personal communication, Analis Ltd, Belgium).

For a 6 year old boy (case $O$, fig 1), total alkaline phosphatase was $403 \mathrm{U} / 1$, bone alkaline phosphatase $310 \mathrm{U} / \mathrm{L}$, liver alkaline phosphatase $40 \mathrm{U} / 1$, intestinal alkaline phosphatase $17 \mathrm{U} / 1$ and intestinal variant alkaline phosphatase $36 \mathrm{U} / 1$.

\section{Discussion}

This family is unique in several aspects. Firstly, agar electrophoresis of alkaline phosphatase showed that there was evidence of an intestinal isoenzyme as well as of an extra band. After analysis with the agarose method the latter was shown to be an intestinal variant isoenzyme. This is a recently discovered
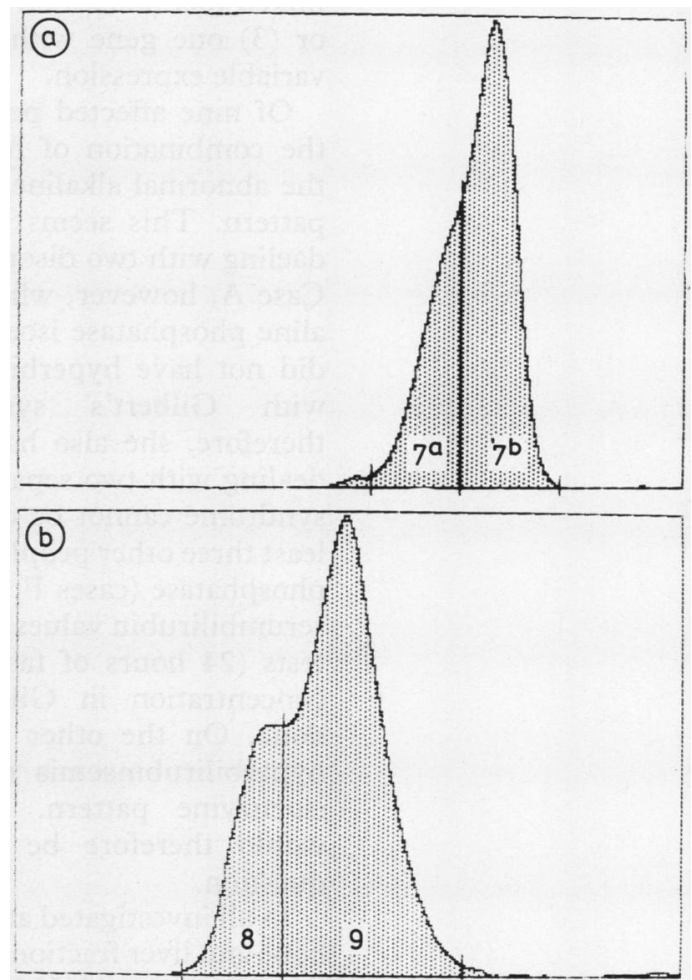

Figure 6 Densitometrical scanning of agarose gel electrophoresis shown in fig 5 with relative fractions ( $\%)$. (a) Above, without neuraminidase preincubation: (7a) bone (33); (7b) liver (67). (b) Below, after neuraminidase preincubation: (8) bone (26); (9) liver (74). Note: preincubation with neuraminidase gives better separation of bone and liver alkaline phosphatase isoenzymes. 
second intestinal isoenzyme, which appears in routine electrophoresis as a bone isoenzyme. ${ }^{21}$ Electrophoretic mobility is not affected after neuraminidase treatment in contrast to bone alkaline phosphatase.

A review of the literature showed nine publications about a familial chronic increased alkaline phosphatase activity. ${ }^{6-14}$ All of those families had an isolated increase in alkaline phosphatase activity. Determination of the alkaline phosphatase isoenzymes in the cited studies showed an increase in either bone, or liver, or intestinal fractions. According to the previous studies a familial increase in alkaline phosphatase activity is a benign disorder-that is, inherited in an autosomal dominant manner. ${ }^{7-911}$ The chromosomal localisation of the encoding gene for alkaline phosphatase is not known, although there have been some speculations about more than three distinct genetic loci. ${ }^{22-24}$

Secondly, apart from the inherited increase in alkaline phosphatase, Gilbert's syndrome was present in this family. Gilbert's syndrome is generally regarded as an autosomal dominant disorder with varying penetration. ${ }^{17}$ Penetrance is reduced in women, perhaps indicating higher normal values in men. Chromosomal localisation is unknown. ${ }^{17}$ The mode of inheritance of the increase in alkaline phosphatase as well as of Gilbert's syndrome in our family fits in with the previously reported data. ${ }^{6-14}$ Possible explanations of the presence of both disorders in this family are (1) two separate genetic disorders with unlinked loci; or (2) two separate genetic disorders with linked loci (alleles on the same chromosome); or (3) one gene with pleiotropic effects and variable expression.

Of nine affected people only three showed the combination of hyperbilirubinaemia and the abnormal alkaline phosphatase isoenzyme pattern. This seems to indicate that we are daeling with two disorders with unlinked loci. Case A, however, who had an abnormal alkaline phosphatase isoenzyme pattern but who did not have hyperbilirubinaemia, has a son with Gilbert's syndrome. Conceivably, therefore, she also has both genes if we are dealing with two separate disorders. Gilbert's syndrome cannot be definitely excluded in at least three other people with abnormal alkaline phosphatase (cases F, K, O) and with normal serumbilirubin values, because no provocation tests (24 hours of fasting increases bilirubin concentration in Gilbert's syndrome) were done. On the other hand, two people with hyperbilirubinaemia did not show a variant isoenzyme pattern. Extended family data would therefore be required to solve the question.

In all investigated adults the sum of absolute bone and liver fractions of alkaline phosphatase was less than $95 \mathrm{U} / \mathrm{l}$, which means that the absolute increase in alkaline phosphatase is caued by intestinal and intestinal variant alkaline phosphatase isoenzymes. These isoenzymes are not normally present in these amounts. The six year old boy (case 10) seemed to have absolute values of intestinal and intes- tinal variant alkaline phosphatase isoenzymes in the same range as the adults in the family. His bone alkaline phosphatase isoenzyme was in the upper normal range for his age.

Although a familial isolated increase in alkaline phosphatase activity is supposed to be rare, it is wise to consider this disorder if an increased alkaline phosphatase is not easily explained by normal growth spurts, pregnancy, bone or hepatobiliary disease, or malignancy. This prevents unnecessary and invasive investigation. ${ }^{9}$

Determination of alkaline phosphatase isoenzymes should probably have a more prominent place in the differential diagnostic process using the available improved biochemical methods.

We thank Mrs E Venekamp-Hookema, Mrs R Prins and M Rademaker for their skilful technical assistance, and Drs A Martens and M Marugg for their assistance.

1 Crofton PM. Biochemistry of alkaline phosphatase isoenzymes. CRC Crit Rev Clin Lab Sci 1982:161-87.

2 Posen S, Lee C, Vines R, Kilham H, Latham S, Keefe JF. Transient hyperphosphatasemia of infancy-an insufficiently recognised syndrome. Clin Chem 1977;23:292-4.

3 Wieme RJ. More on transient hyperphosphatasemia, an insufficiently recognized syndrome. Clin Chem 1978;24:520-2

4 Nathan E. Transient hyperphosphatasemia of infancy. Acta Paediatr Scand 1980;69:236-8.

5 De Vito Jr GA. Transient elevation of alkaline phosphatase possibly related to trimethoprim-sulfamethoxazole therapy. J Paediatr 1982;100:998-9.

6 Abassi V, Colon AR, Schwartz RA. Benign elevation of serum alkaline phosphatase, transient and persistent variety. Clin Paediatr 1984;23:336-7.

7 Wilson JW. Inherited elevation of alkaline phosphatase activity in the absence of disease. $N$ Engl J Med 1979;301:983-4.

8 McEvoy M, Skarbanek P, Wright E, Powell D, McDonagh B. Family with raised serum alkaline phosphatase activity in the absence of disease. Br Med J 1981;282:1272.

9 Ducobu J, Dupont P. Inherited raised alkaline phosphatase activity in the absence of disease. Lancet 1981;i:1372-3.

10 Parker PH, Ghishan FK. Asymptomatic familial elevation of serum alkaline phosphatase levels. Am J Dis Child 1980;134:1094-5.

11 Cirera Nogueras L, Vivancos Lleida J, Salazar Badia M, et al. Raised serum alkaline phosphatase activity in one family. Arch Intern Med 1982;142:188-9.

12 Kruse K. Inherited isolated hyperphosphatasemia. Acta Paediatr Scand 1983;72:833-5.

13 Rapado A, Esbrit P. Hiperfosfatasia asintomatica familiar por elevacion de la isoenzima intestinal. Rev Clin Esp 1984;173:53-4.

14 Lewis JM. Persistent isolated hyperphosphatasemia Southern Med J 1987;80:788-9.

15 Gilbert A, Lerebouillet P. La cholemie simple familiale. Semaine Medicale 1901;21:241-3.

16 Schmid R. Hyperbilirubinemia. In: Stanbury JB Wijngaarden JB, Fredrickson DS, ed. 3rd ed. The metabolic basis of inherited disease. New York: McGraw-Hill 1972:1141-78.

17 Billing BH. Bilirubin metabolism. In: Schiff L. Diseases of the liver. 6th ed. Philadelphia: JB Lippincott, 1987:113-7.

18 Sundblad L, Wallin-Nilsson M, Brohult J. Characterization of alkaline phosphatase isoenzymes in serum by agar gel electrophoresis. Clin Chim Acta 1973;15:219-23.

19 Robson EB, Harris H. Further studies on the genetics of placental alkaline phosphatase. Ann Hum Gene 1967;30:219-23.

20 Moss DW, Edwards RK. Improved electrophoretic resolution of bone and liver Alkaline Phosphatase resulting from partial digestion with neuraminidase. Clin Chim Acto 1984;143:177-82.

21 Van Hoof VO, Lepoutre LG, Hoylaerts MF, Chevingné $R$ De Broe ME. Improved agarose electrophoretic method for separating alkaline phosphatase isoenzymes in serum. Clin Chem 1988;34:1857-62.

22 Mueller HD, Leung H, Stinson RA. Different genes code for alkaline phosphatases from human fetal and adult
intestine. Biochem Biophys Res Commun 1985;126:427-33.

23 McKusick VA. Mendelian inheritance in man. Catalogs of autosomal dominant, autosommal recessive and $x$-linked phenotypes. Baltimore: John Hopkins University Press, 1988 .

24 Millan JL, Eriksson A, Stigband T. A possible new locus of alkaline phosphatase expressed in human testis. Hum Genet 1982;62:293-5. 